\title{
Otorhinolaryngology - not just tonsils and grommets: Insights into the ENT scene in South Africa
}

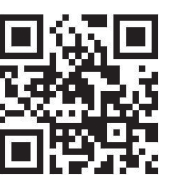

Ear, nose and throat (ENT) surgery may be the oldest surgical specialty, but it certainly has not lost its lustre with age. It encompasses a wide variety of pathology, spanning all age groups regardless of gender, ethnicity or socio-economic status. The field of ENT (otorhinolaryngology) includes a broad range of procedures, often using advanced technology both at the bedside and in theatre. Recent decades have seen the discipline expand to include subspecialties like head and neck surgery, neuro-otology, anterior and lateral skull base surgery, allergology and paediatrics.

In South Africa (SA), where it is very apparent that there are economic barriers to diagnosis and treatment, we are faced with the co-existence of the developing and developed world. Health budgets, poor infrastructure and shortage of medical staff are some of the challenges regularly encountered. ${ }^{[1]}$ Furthermore, there are individual clinicians and dedicated units who strive for the best outcomes by pioneering ground-breaking surgeries, regardless of limitations in resources - including head and neck cancer units using free flaps, endoscopic skull-base surgery using neuro-navigation and cochlear implant programmes, to name a few.

Almost $20 \%$ of general practitioner (GP) visits involve complaints arising from the ears, nose or throat. In the paediatric population, this figure rises to around 50\%.[2] As of 31 March 2012, 38236 medical practitioners are registered with the Health Professions Council of South Africa (HPCSA), of whom only $0.9 \%$ are ENT specialists (344 practitioners). ${ }^{[3,4]}$ In addition, almost $70 \%$ of the 
44 million South Africans who utilise the public health sector are serviced by less than 35 full-time ENT specialists. Consequently, the majority of ENT problems are managed by non-ENT specialists.

The global health stage is dominated by communicable diseases like HIV and tuberculosis (TB). Many argue that diseases like hearing impairment and head and neck cancers receive less attention, despite being major public health concerns. In SA, communicable and noncommunicable ENT diseases are interlinked owing largely to the high prevalence of HIV and the increasing problem of multi-drug resistant (MDR) TB, together with the existing socio-economic challenges.

SA has the highest number of HIV-infected individuals in any country, with the highest prevalence among people between the ages of 15 and 49 years. ${ }^{[5]} \mathrm{Up}$ to $100 \%$ of HIV-positive patients will present with head and neck manifestations. ${ }^{[6]}$ Oral candidiasis and rhinosinusitis were traditionally documented as the most common ENT manifestations of $\mathrm{HIV}^{[7]}$ However, with the institution of antiretroviral (ARV) rollout programmes, patients may present differently. This has been suggested by Tshifularo et al. ${ }^{[8]}$ who found adenoid pathology, cervical lymphadenopathy and chronic suppurative otitis media to be the top three manifestations at Steve Biko Academic Hospital. While this may imply a change, it could be multi-factorial, rather than solely drug-related effects. More research is therefore needed from patients on long-term ARV therapy.

According to the World Health Organization (WHO), hearing impairment is the greatest sensory deficit globally. There are 360 million people worldwide suffering from disabling hearing loss ( $>40 \mathrm{db}$ ), more than half of whom live in low- and middleincome countries ${ }^{[9]}$ Of greater concern is that these people may not be identified as having hearing loss, because there are not enough screening programmes in place. ${ }^{[9]}$

Perhaps most tragic, $50 \%$ of all causes of hearing loss are preventable causes that can be alleviated by early prevention techniques, including screening programmes and early definitive management strategies. ${ }^{[9]}$ HIV-positive people have an increased risk of developing hearing loss - primarily from the disease (non-iatrogenic), from opportunistic infections, or from the effects of drugs related to treatment. ${ }^{[10]}$ Antiretroviral therapy (ART), alone or in combination with other treatments, could add to the overall toxic effect on hearing. ${ }^{[10]}$ In SA, where the increase in MDR-TB has been linked to the HIV epidemic, patients on WHO-recommended MDR-TB second-line drug therapy are more likely to have nephrotoxicity and ototoxicity, the latter being permanent. ${ }^{[11]}$ Some individuals may develop sudden profound sensorineural hearing loss following a single dose of aminoglycoside, highlighting the need to use it with caution even in routine medical practice. Harris et al. ${ }^{[11]}$ found that $57 \%$ of MDRTB patients developed high frequency hearing loss, and that $70 \%$ of HIV-positive patients were more likely to develop hearing loss than HIV-negative patients on MDR-TB therapy. As a consequence, HIV and TB co-infected individuals are a high-risk population for hearing loss, and without awareness and intervention, may constitute a new deaf community in sub-Saharan Africa (SSA) - a population that will require audiological and ENT services. ${ }^{[1]}$

Despite SA being the most advantaged in Africa regarding access to audiology services, hearing aids and ENT interventions, Fagan and Jacobs ${ }^{[12]}$ found that our country still only fulfilled less than half of the required service ratios compared with the UK. SA does not have adequate screening programmes for children, the elderly or high-risk populations.

The global burden of illness from chronic suppurative otitis media (chronic active otitis media) involves 65 - 330 million individuals with otorrhoea, $60 \%$ of whom suffer from significant hearing impairment. ${ }^{[13]}$ Prevalence varies in different population groups, with local prevalence rates in SSA ranging between 0.4 and $4.2 \%-50 \%$ of these cases presenting in children under 10 years. ${ }^{[14]}$

Otorrhoea is treated at a primary care level in SA, and patients are generally referred to specialists only after treatment failure. Tiedt et al.$^{[14]}$ and Meyer et al. ${ }^{[15]}$ undertaking local studies at different centres, highlight the delay in patients being referred to ENT services. In children who suffer associated hearing loss this delay has a negative impact on school performance. ${ }^{[14]}$

In addition, Loock ${ }^{[16]}$ and the aforementioned two local ENT departments investigated the microbial spectrum of otorrhoea in their respective communities. ${ }^{[14-17]}$ All of the local studies have identified Proteus mirabilis and Pseudomonas aeruginosa as the most prevalent pathogens. This differs from findings elsewhere in the world. Guidelines for the management of otitis media, aimed at a primary healthcare level, were released by the the National Department of Health in 2012. ${ }^{[18]}$ Although very comprehensive, the recommended antimicrobials (topical or systemic), including oral amoxicillin, for chronic otorrhoea (non-responders) do not cover the majority of organisms encountered. However, topical fluoroquinolone eardrops with ear mopping covers $95 \%$ of organisms cultured and is not ototoxic. Should the first-line treatment fail then an antibiotic group with Gram-negative cover should be considered. ${ }^{[15]}$

Patient profiles, disease projection and response to treatment all vary according to the location and population. In many spheres of medicine, we rely heavily on international sources, often adapting protocols and guidelines for local use. The above findings emphasise the need to promote local research.

Ideally, smaller surgical disciplines like ENT should capture data at local institutions and keep national registries for specific diseases. In doing so, the burdens of disease can be better managed, and local databases can also be used for auditing local services. Research should be integrated - across institutions, between disciplines, across departments and beyond economic barriers into the private sector - where pioneering work often goes unpublished. Comparative assessments of local versus international findings are also necessary, thereby facilitating a global perspective for SA ENT centres that will enable them to improve and compete with world leaders in the field.

Head and neck squamous cell cancer (HNSCC) is the sixth most common malignancy and the eighth leading cause of cancer death worldwide. ${ }^{[19]}$ The incidence in the developing world is on the rise attributable to increased life expectancy from communicable disease control, excess tobacco and alcohol consumption (risk factors for head and neck cancers) and HIV. ${ }^{[1]}$ Treatment outcomes for patients treated in developing world versus developed world countries are quite different. Farmer et al. ${ }^{[20]}$ found an association between cancer fatality rates and national income - the cancer case fatality rate in low-income countries is $75 \%$, as opposed to $45 \%$ in high-income countries.

HNSCC is also the third most common head and neck malignancy for patients with HIV (after Kaposi's sarcoma and non-Hodgkin's lymphoma). ${ }^{[21]}$ Although not an AIDS-defining illness, HNSCC is seen in excess among HIV-infected individuals, and is sometimes referred to an 'opportunistic' cancer. Patients with a suspicious persistent head and neck lesion should always be referred for further investigation.

In addition, there is growing evidence that confirms the causative relationship between oncogenic human papillomavirus (HPV) and oropharyngeal squamous carcinoma in the general population. The prevalence of HPV in oropharyngeal cancer, especially tonsillar cancer, is as high as $90 \%$. HPV type 16 is most commonly involved. An unexpected and positive finding in the literature is HPV-positive 
HNSCC being associated with high-stage tumours, but actually having better survival. ${ }^{[21]}$

There are many challenges that face ENT specialists - as clinicians who value contribution to individual patients, and in the broader field of population health. Issues highlighted in this editorial help give insight into the current ENT perspective, notwithstanding other important topics that have not been addressed.

The SA Society of Otorhinolaryngology, Head and Neck Surgery is well established and includes private and public specialists, as well as registrars in training. Also established is the National Registrar Society, a subsidiary body that is managed independently by elected registrars and includes a national database and interactive website. Both divisions are actively committed to maintaining a high standard of training.

\section{S Peer}

Department of Otorhinolaryngology, University of Cape Town, South Africa, and Clinical Research Fellow, Department of ENT, SickKids Hospital, Toronto, Canada

\section{Corresponding author: S Peer (shaziapeer@gmail.com)}

1. Fagan JJ. Developing world ENT: A global responsibility. J Laryngol Otol 2012;126(6):544-547. [http:// dx.doi.org/10.1017/S0022215112000345]

2. Clamp PJ, Gunasekaran S, Pothier DD, Saunders MW. ENT in general practice: Training, experience and referral rates. J Laryngol Otol 2007;121(6):580-583. [http://dx.doi.org/10.1017/S0022215106003495] 3. HPCSA. iRegister. http:///iregister.hpcsa.co.za/RegisterSearch.aspx (accessed 4 June 2013)

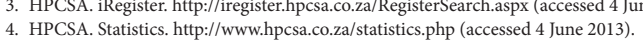

4. HPCSA. Statistics. http://www.hpcsa.co.za/statistics.php (accessed 4 June 2013).
5. UNAIDS. UNAIDS report on the global epidemic. Geneva: UNAIDS, 2010. http://www.unaids.org/ 5. UNAIDS. UNAIDS report on the global epidemic. Gen
globalreport/global_report.htm (accessed 4 June 2013).
6. Tami TA, Hairston JA. HIV and otolaryngology. In: Gleeson M, ed. Scott-Brown's Otorhinolaryngology, Head and Neck Surgery. London: Hodder Arnold, 2008:238-250.

7. Lubbe DE. HIV and ENT. CME 2004;22:250-253.

8. Tshifularo M, Govender L, Monama G. Otolaryngological, head and neck manifestations in HIV-infected patients seen at Steve Biko Academic Hospital in Pretoria, South Africa. S Afr Med J 2013;103(7):464-

466. [http://dx/doi.org/10.7196/SAMJ.6786]
9. World Health Organization. Deafness and hearing loss. Geneva: WHO, 2013. http://www.who.int/ World Health Organization. Deafness and hearing loss. Geneva:
mediacentre/factsheets/fs300/en/index.html (accessed 12 June 2013).

10. Khoza-Shangase K. Is there a need for ototoxicity monitoring in patients with HIV/AIDS? African Journal of Pharmacy \& Pharmacology 2010;4(9):574-579.

11. Harris, T, Bardien S, Schaaf HS, et al. Aminoglycoside-induced hearing loss in HIV-positive and HIVnegative multidrug-resistant tuberculosis patients. S Afr Med J 2012;102(6):363-366.

12. Fagan JJ, Jacobs M. Survey of ENT services in Africa: Need for a comprehensive intervention. Global Health Action 2009;2:e1-e7. [http://dx.doi.org/10.3402/gha.v2i0.1932]

13. WHO. Chronic suppurative otitis media. Burden of illness and management options. Geneva: WHO, 2004. http://www.who.int/pbd/deafness/activities/hearing_care/otitis_media.pdf (accessed 4 June 2013).

14. Tiedt NJ, Butler IR, Hallbauer UM, et al. Paediatric chronic suppurative otitis media in the Free State Province: Clinical and audiological features. S Afr Med J 2013;103(7):467-470. [http://dx/doi. org/10.7196/SAMJ.6636]

15. Meyer E, Whitelaw A, Edkins O, Fagan JJ. Chronic otorrhoea: Spectrum of microorganisms and antibiotic sensitivity in a South African cohort. S Afr Med J 2013;103(7):471-473. [http://dx/doi. org/10.7196/SAMJ.6066]

16. Loock JW. A randomised controlled trial of active chronic otitis media comparing courses of eardrops v. one-off topical treatments suitable for primary, secondary and tertiary healthcare settings. Clin Otolaryngol 2012;37(4):261-270. [http://dx.doi.org/10.1111/j.1749-4486.2012.02532.x]

17. Seedat RY, Bester A, Möller E, et al. Bacteriology of chronic suppurative otitis media in the era of ototopical quinolone use. 42nd Annual Congress of the South African Society of OtorhinolaryngologyHead and Neck Surgery. Cape Town, 8-11 October 2006.

18. South African Department of Health. National Guidelines for the Management of Otitis Media. Pretoria: DoH. http://www.doh.gov.za/docs/policy/2012/hearingguide.pdf (accessed 4 June 2013).

19. Parkin DM, Bray F, Ferlay J, Pisani P. Global cancer statistics, 2002. CA Cancer J Clin 2005;55(2):74108. [http://dx.doi.org/10.3322/canjclin.55.2.74]

20. Farmer $P$, Frenk J, Knaul FM, et al. Expansion of cancer care and control in countries of low and middle income: A call to action. Lancet 2010;376(9747):1186-1193. [http://dx.doi.org/10.1016/S0140-6736(10)61152-X]

21. McLemore ML, Haigentz Jr M, Smith RV, et al. Head and neck squamous cell carcinomas in HIV-positive patients: A preliminary investigation of viral associations. Head Neck Pathol 2010;4(2):97-105. [http://
(a) dx.doi.org/10.1007/s12105-010-0171-9]

S Afr Med J 2013;103(7):455-457. DOI:10.7196/SAMJ.7121 